\title{
Cavitation Detection in Variable Speed Pump by Analyzing the Acoustic and Vibration Spectrums
}

\author{
Ashraf Kotb, Abdulaziz Morgan Abdulaziz \\ Department of Mechanical Power Engineering, Faculty of Engineering, Ain Shams University, Cairo, Egypt \\ Email: Ashraf Ibrahim@eng.asu.edu.eg, Abdelaziz Morgan@eng.asu.edu.eg
}

Received 24 August 2015; accepted 27 October 2015; published 30 October 2015

Copyright (C) 2015 by authors and Scientific Research Publishing Inc.

This work is licensed under the Creative Commons Attribution International License (CC BY). http://creativecommons.org/licenses/by/4.0/ c) (7) Open Access

\begin{abstract}
Cavitation in pumps must be detected and prevented. The present work is an attempt to use the simultaneous measurements of vibration and sound for variable speed pump to detect cavitation. It is an attempt to declare the relationship between the vibration and sound for the same discharge of $780 \mathrm{~L} / \mathrm{h}$ and $\mathrm{NPSH}_{\mathrm{A}}$ of 0.754 at variable speeds of $1476 \mathrm{rpm}, 1644 \mathrm{rpm}, 1932 \mathrm{rpm}, 2190$ rpm, $2466 \mathrm{rpm}$, and $2682 \mathrm{rpm}$. Results showed that: the occurrence of cavitation depends on the rotational speed, and the sound signals in both no cavitation and cavitation conditions appear in random manner. While, surveying the vibration and sound spectrums at the second, third, and fourth blade passing frequencies reveals no indications or phenomenon associated with the cavitation at variable speeds. It is recommended to survey the vibration spectra at the rotational and blade passing frequencies simultaneously as a detection unique method of cavitation.
\end{abstract}

\section{Keywords}

Centrifugal Pump, Cavitation, Vibration, Blade Passing Frequency, Variable Speed

\section{Introduction}

Installation of pump is often sized to cope with a highest predicted discharge, which, may never occurred. This over sizing is used in industry, and leads to wasted energy and damage to parts of the pump. Speed regulation is one of the best methods to control the pump discharge, it has many benefits include; energy savings, reliability enhancement, simple piping and connections, soft start and stop, and reduced maintenance. Lowering the pump rotational speed reduces the hydraulic forces on the impeller approximately with the square of the rotational speed. These hydraulic forces are generated as a result of the pressure profile inside the pump casing, and car- 
ried by the bearings. Therefore, reducing the rotational speed increases the life time of the bearings which is inversely proportional to the $7^{\text {th }}$ power of the rotational speed. Vibration and noise are reduced and seal life is increased providing the duty point remains within the allowable operating range as a result of lowering the rotational speed.

For a pump rotates at any running speed, the liquid entering the impeller eye turns and is split into streams by the leading edges of the impeller blades, this splitting locally drops the fluid pressure below that in the suction pipe. This situation becomes more dangerous when the incoming liquid is at a pressure with small margin above the vapor pressure corresponding to the liquid temperature. Cavities or bubbles of vapor create along the impeller blades just behind the inlet edges, and this is well known as cavitation in pumps. Cavitation has undesirable effects; erode the blade surface, shorten seal and bearing life as a result of increased noise and vibration, choke the flow passages in the impeller, worsen the pump performance.

The pump manufacturer provides the pressure in the pump suction exceeds the vapor pressure to prevent cavitation. This value is known as the required net positive suction heat $\mathrm{NPSH}_{\mathrm{R}}$ which is a characteristic of the pump design and manufacturing. $\mathrm{NPSH}_{\mathrm{R}}$ depends on the pump rotational speed as follows; $\mathrm{NPSH}_{\mathrm{R}} \propto N^{2}$. It should be noted however that at very low speeds there is a minimum $\mathrm{NPSH}_{\mathrm{R}}$ plateau, $\mathrm{NPSH}_{\mathrm{R}}$ does not tend to zero at zero speed. It is therefore essential to carefully consider $\mathrm{NPSH}_{\mathrm{R}}$ in variable speed pumps. On the other hand, the pressure exceeds the vapor pressure in the pumping system is expressed as the available net positive suction head $\mathrm{NPSH}_{\mathrm{A}}$ and it is a characteristic of the system. Manufacturers and industry BPMA, define the onset of cavitation as the value of $\mathrm{NPSH}_{\mathrm{R}}$ when there is a head drop of $3 \%$ compared with the head for cavitation free performance. Al-Arabi et al. [1] visually observed cavitation develops at the state where the pump head drops by $3 \%$, but the onset of cavitation appears long before the pump performance is affected. At 3\% drop of total head severe cavitation is highly likely and design on the basis of that could cause an erroneous prediction estimate of pump performance.

There are several methods for detecting the incipient of cavitation; determination of the net positive suction head at a constant speed and flowrate, visualization of the inlet flow at the impeller, paint erosion on impeller blades, static pressure measurement in the flow, vibration and acoustic measurements. Vibration and net positive suction head measurements are widely applied, and they have been studied, see for instance [2].

Cavitation detection by acoustic measurements is based on understanding the sources of the generated noise from the pump [3]. Mechanical sources of noise include vibration of pump components as a result of; pressure variations through the pump flow passages, defective components such as impeller, seal rubs, bearings, unbalanced impellers, improper installation of pump driver and misalignment. Hydraulic sources of noise include both the transient and instationarity of flow causes; Transients; during the startup and shutdown of the pump, opening or closing of servicing valves may cause severe pressure waves (water hammer) with high intensity of noise levels. Instationarity of flow; as a result of asymmetric outgoing flow of the impeller due to secondary flows in the rotating impeller, number of vanes and thickness which cause a notch in the wake flow, guide vanes and tongue in the pump casing. The instationarity of flow is a combination of both the effect of pressure pulsations and turbulence. Pressure pulsations depend on the design of impeller, diffuser, operating parameters, and the distance between impeller and the volute tongue. Turbulence induces vortices and wakes in the clearance between the impeller vane tips and the diffuser or volute lips. Pressure pulsations produced in this way impinge on the impeller and result in shaft vibrations and noise over a wide frequency range.

As the vibration measurement is used to detect cavitation, it is important to differentiate between the roots and causes of vibration in pumps. Ravindra et al. [3] attributed the root of vibrations in pumps to mechanical, hydraulic, or peripheral aspects. It is easy to trace the mechanical causes but it becomes difficult to trace hydraulic causes which make diagnostic very complex. The mechanical causes of vibration include; unbalanced rotating components, damaged impellers and non-concentric shaft sleeves, bent or warped shaft, pump and driver misalignment, pipe strain (either by design or as a result of thermal growth), inadequacy of foundations or poorly designed foundations, thermal growth of various components, especially shafts, rubbing parts, worn or loose bearings, loose parts, loosely held holding down bolts, damaged parts. The hydraulic causes of vibration include; operating pump at other than best efficiency point, vaporization of the product, impeller vane running too close to the pump volute tongue, internal recirculation, air entrapment into the system through vortexing etc., turbulence in the system (non-laminar flow), water hammer. The peripheral causes of vibration include; harmonic vibration from nearby equipment or drivers, operating the pump at a critical speed, temporary seizing of seal faces. 
Previous work includes many attempts to clarify the detection of cavitation by measuring vibration and/or acoustic levels. Alfayez et al. [4] studied two stages "David Brown” $60 \mathrm{KW}$ centrifugal pump (Model DB22) with a maximum capacity of $204 \mathrm{~m}^{3} / \mathrm{h}$ at an efficiency of $70.6 \%$. The results from acoustic emission analysis have shown a clear relationship between AE activity measured from the pump casing, suction and discharge pipes, and incipient cavitation.

Cernetic et al. [5] studied two centrifugal pumps; pump A made of metal alloy with 6 blades closed impeller. Pump B made of plastic material with 6 blades semi-open impeller. The results showed that; each pump has specific characteristics, different spectra, level of noise and vibration, and different discrete frequency. Detection method should be determined for each pump individually. Also, the difference in the noise and vibration levels between cavitating and non-cavitating conditions are above $10 \mathrm{db}$.

Cernetic and Cudina [6] showed that the cavitation noise is usually recorded mainly as noise of higher frequency range. This finding is somehow contradictious because it is known that cavitation cause noise also in lower frequency range. It shows that cavitation produces not only noise, but also strong vibration of the pumping system at lower frequency range, which excites air in surroundings and is also heard as noise. These vibrations can be measured as noise during the operation of the pump.

Albarik et al. [7] studied single suction, single stage, end/top discharge, closed impeller and closed-coupled centrifugal pump, which can deliver water at a rate of up to $30 \mathrm{~m}^{3} / \mathrm{h}$ at a head of up to $55 \mathrm{~m}$. It is driven by a three-phase electric motor running at $2900 \mathrm{rpm}$ on $9.5 \mathrm{~A}$ at $380-400 \mathrm{~V}$ (nominal $4 \mathrm{~kW}$ ). The results showed that the vibration level increases with increased of flow rate and the pump faults may then be identified using these data/features.

Amit [8] used single stage horizontal and diffuser type pump with impeller having 8 vanes and diffuser having 7 vanes. Radial clearances of the order $6.8 \mathrm{~mm}, 3.7 \mathrm{~mm}, 1.5 \mathrm{~mm}$ has been used during the experimentation. Amit found that; vibration is dominating at the fundamental frequency. The overall level is low at low flow rates and high at higher flow rates and is minimum at maximum radial clearance. Noise is dominating at impeller vane passing frequency. The overall noise level is low at low flow rates and high at higher flow rates. Moreover the overall noise level is minimum at maximum radial clearance.

Shrikumar et al. [9] for $550 \mathrm{~kW}$ pumping set runs at $2535 \mathrm{rpm}$, found a difference of $18 \mathrm{~dB}$ in the noise level before the incipient of cavitation and after fully developed cavitation at the discrete frequency of $175 \mathrm{~Hz}$ for pump discharge from $0.12 \mathrm{~m}^{3} / \mathrm{s}$ to $0.075 \mathrm{~m}^{3} / \mathrm{s}$.

Osama et al. [10] carried out experiments based on pump delivers $350 \mathrm{~L} / \mathrm{min}$ of water under pressure of 10 bar when the driving motor operates at $2900 \mathrm{rpm}$. The experiment was carried out based on a same pump casing but three different impellers. The first impeller is a healthy one whereas the second and third have inlet vane fault and exit vane fault respectively. The fruitful results that; vibration signals consist of two contents. The discrete content from mechanical and hydraulic pulsations is more deterministic whereas the wideband content from flow turbulences and cavitation is more random in nature.

Ramadevi [11] investigated the cavitation detection using discrete wavelet transform by classifying the pump vibration signal. Vibration signal acquired from centrifugal pump cavitation test rig carry more information about the cavitation classes. Two classes has been defined namely, no cavitation class and developed cavitation class. This method uses the deviation from zero mean value of detailed components of wavelet coefficients, obtained from five level decomposition of vibration signal to detect the signal belongs to normal class or cavitation class in centrifugal pump. The main advantage of this proposed algorithm is it requires no training. In addition to this advantage a more robust results show that this algorithm has better detection response.

Zhaoli et al. [12] proposed a novel method to detect cavitation based on active ultrasonic flow field velocity measurement. The effect of liquid cavitation on the ultrasonic propagation velocity was verified by the experimental study. The power of the demodulated signal, being almost zero when the fluid was still, clearly increased along with fluid motion. Furthermore, a sudden increment in the power and pulse characteristics appeared during cavitation inception.

The accuracy of the proposed cavitation recognition method is over $98 \%$, which is comparable to the performance of the acoustic method using a hydrophone. In addition, the ultrasonic transducers in the proposed method are easy to be installed on the outer surface of the pipeline. This nonintrusive installation method is more convenient than the installation methods that use pressure and hydroacoustic signal analysis in some engineering applications.

The present work is an attempt to use the simultaneous measurements of vibration and sound for variable 
speed pump to detect cavitation. It is an attempt to declare the relationship between the vibration and sound for the same discharge at variable speeds.

\section{Experimental Setup}

The experiments were carried out on a setup especially designed and built for cavitation studies in pumps, BMPA [13]. The setup is built in Research Studies Laboratory-Department of Mechanical Power Engineering-Faculty of Engineering-Ain Shams University. The experimental setup is in accordance with ISO 3555 and BS EN ISO 9906/2000 specifications for acceptance tests for centrifugal, mixed flow and axial pumps Class B tests and it is shown schematically in Figure 1. It is composed from a closed loop water circuit containing pump, tank, connecting PVC pipes, regulating valves.

Since the cavitation is dependent on the temperature (vapor pressure) of the fluid, then the tank is fitted by means of cooling system to assure constant temperature during all measurements. Acoustic and vibration measurements are sensitive to any external and surrounding sources, for this reason; using PVC pipes with rubber rings and allocation of the regulating valves far from the pump minimize the interfering with the pump measurements, and all measurements were carried out in times where any background noise is excluded. The pump is centrifugal and it has an impeller of closed type with 6 blades and its suction specific speed at rated rotational speed is $5130 \mathrm{rpm}$.

\section{Instrumentation}

The test rig is provided with a set of measuring instrumentation for different variables. The pump rotational speed is precisely controlled with an electronic inverter within $0.1 \mathrm{~Hz}$ resolution. The upstream and downstream pressures of the pump are measured by pre-calibrated dial gauges with additional pressure transducer; Keller Pa21SR, to record the downstream pressure. The pressure transducer accuracy is \pm 0.5 of full scale. Pump discharge is measured by a pre-calibrated electromagnetic flow meter. The maximum uncertainty in measuring the pump discharge as repeated 14 times for each test case is $0.5 \%$. The acoustic measurements are made by GRAS free-field microphone type $46 \mathrm{AE}$, place $50 \mathrm{~cm}$ from the pump in the plane parallel to the pump axis. The microphone band width is $3.5: 20,000 \mathrm{~Hz}$ with uncertainty of $\pm 0.06 \mathrm{~dB}$ at the test frequency $250 \mathrm{~Hz}$. Measurements of sound are taken with $52.2 \mathrm{k}$ sample at one second. The vibration acceleration measurements are made by 352C33 ICP ® accelerometer. The accelerometer band width is $0.3-15,000 \mathrm{~Hz}$ with maximum deviation of $\pm 2.5 \%$. Since the vibration due to cavitation is the only main concern of this study, therefore the accelerometer is mounted at the suction port in the radial direction.

All measurements are acquired to a PC via the very specialized NI 9234 data acquisition card. Data acquisition is performed by an NI 9234 card of 4 channels, 24 bits with amplifying capability. The sampling frequency

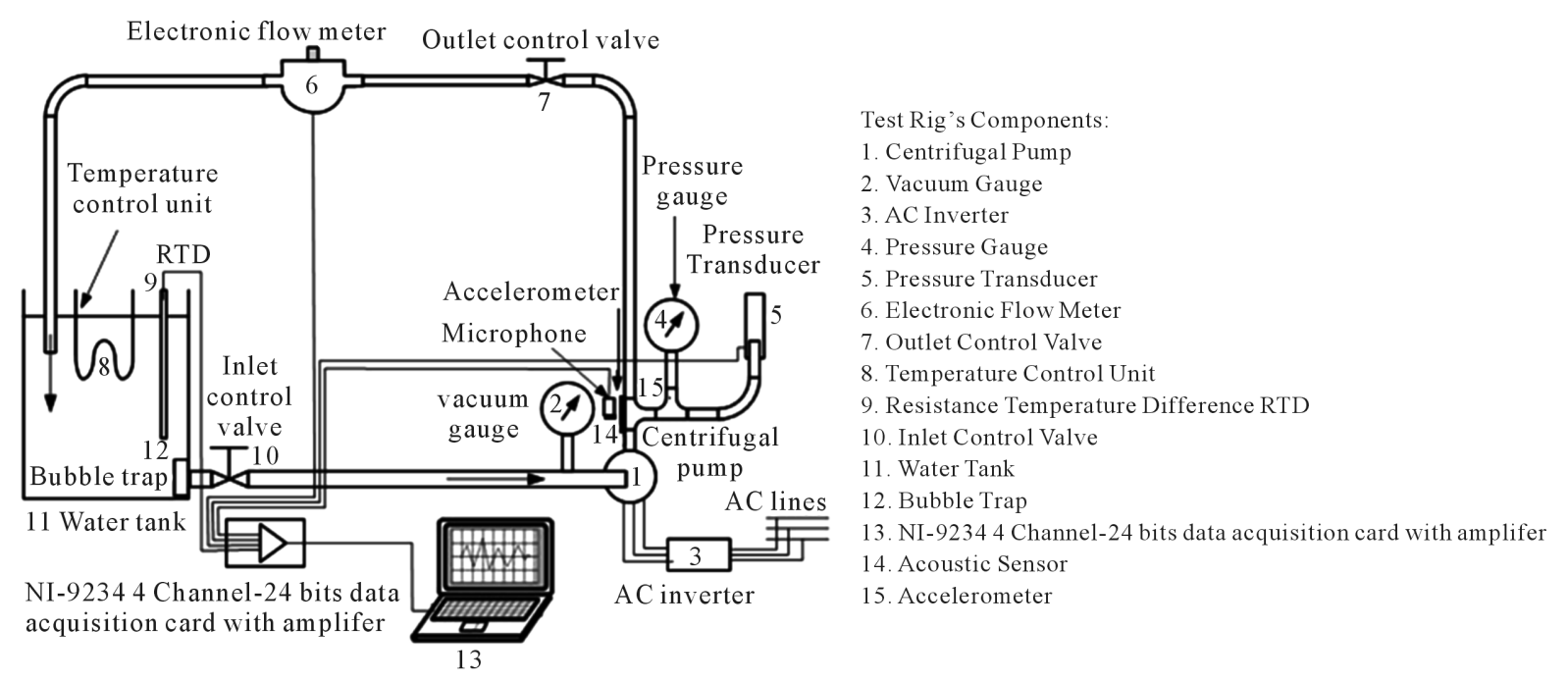

Figure 1. Schematic of experimental test rig. 
is $52.2 \mathrm{kHz}$ for each channel. LabView software is used to simultaneously record the mass flow rate, downstream pressure, sound levels, and vibration acceleration. The software performs Fast Fourier Transform calculations. The measurements are repeated 30 times for the same run and the scheme of peak-hold is applied. Meanwhile the instantaneous volume flow rate and the downstream pressure are continuously acquired and averaged for the 30 second run. All measurements are, therefore, taken simultaneously. The raw and processed data are then stored in an Excel file sheet.

\section{Results and Discussion}

The present work considers six different values of rotational speed of 1476 rpm, 1644 rpm, 1932 rpm, 2190 rpm, $2466 \mathrm{rpm}$, and $2682 \mathrm{rpm}$, the rotational speed is precisely controlled with the electronic inverter. The pump discharge is modulated and kept in the range of $780 \mathrm{~L} / \mathrm{h} \pm 5 \mathrm{~L} / \mathrm{h}$ for all the values of rotational speeds, the adjustment of the pump discharge by a pre-calibrated electromagnetic flow meter is achieved by trials and errors.

Since the available net positive suction head $\mathrm{NPHS}_{\mathrm{A}}$ is based on the vapor pressure at the corresponding fluid temperature, suction pressure which is modulated by the control valve upstream the pump inlet and measured by pre-calibrated dial gauges and set to be $650 \mathrm{mmHg}$ "vacuum", and the velocity of flow through the suction line. Then the value of the available net positive suction head is calculated and it is found to be $0.754 \mathrm{~m}$ for all values of the rotational speed.

To assign the cavitation status for each rotational speed, the affinity laws are used to predict the required net positive suction head at the rotational speeds, while the manufacturer's data at $2850 \mathrm{rpm}$ is considered as the baseline data. Figure 2 shows the calculated required net positive suction head for different rotational speeds at variable pump discharge.

From Figure 2, at the pump discharge of $780 \mathrm{~L} / \mathrm{h}$ one can deduce the cavitation status for each rotational speed as illustrated in Table 1.

The conditions at rotational speeds of $1476 \mathrm{rpm}, 1644 \mathrm{rpm}$, and $1932 \mathrm{rpm}$ are normal pump operation with no cavitation, while for rotational speeds of $2190 \mathrm{rpm}, 2466 \mathrm{rpm}$, and $2682 \mathrm{rpm}$ the pump is subjected to cavitation. Therefore, the cavitation strength (\%) is calculated for each cavitation condition by the following equation:

$$
\xi=100 \times \frac{\mathrm{NPSH}_{\mathrm{R}}-\mathrm{NPSH}_{\mathrm{A}}}{\mathrm{NPSH}_{\mathrm{R}}}
$$

The cavitation strength values are $10.24 \%, 58.10 \%$, and $40.63 \%$ for the rotational speeds of $2190 \mathrm{rpm}, 2466$ rpm, and $2682 \mathrm{rpm}$ respectively. Therefore the studied conditions is divided into two groups, group 1 represents normal operation (no cavitation) of the pump at $1476 \mathrm{rpm}, 1644 \mathrm{rpm}$, and $1932 \mathrm{rpm}$. While group 2 represents pump operates at cavitation at $2190 \mathrm{rpm}, 2466 \mathrm{rpm}$, and $2682 \mathrm{rpm}$ (cavitation).

Figure 3(a), Figure 3(b), and Figure 3(c) shows the root mean square for both the vibration acceleration and

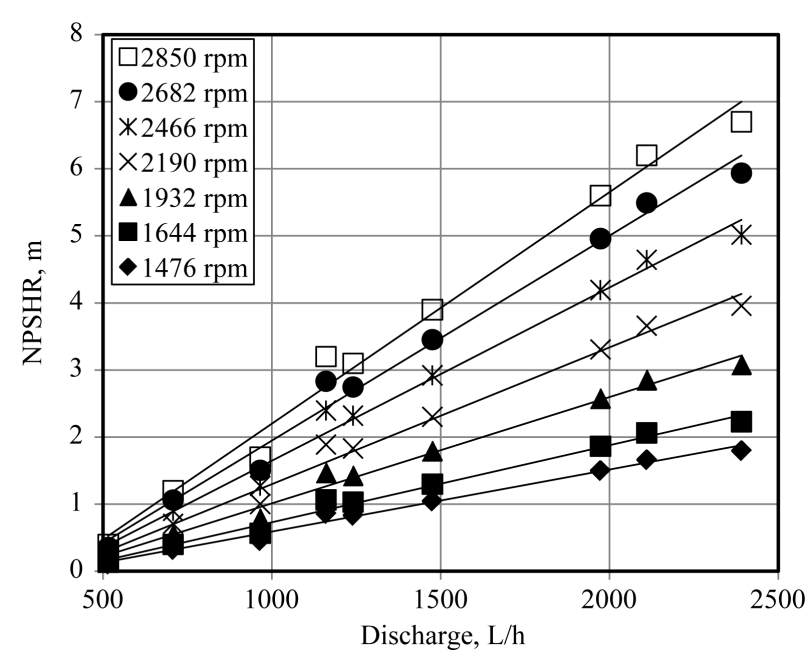

Figure 2. Required net positive suction head at variable pump discharge at different rotational speeds. 
Table 1. Status of the pump at each rotational speed.

\begin{tabular}{ccccc}
\hline Rotational Speed, rpm & $\mathrm{NPSH}_{\mathrm{A}}, \mathrm{m}$ & $\mathrm{NPSH}_{\mathrm{R}}, \mathrm{m}$ Figure 2 & Cavitation Status & $\xi, \%$ \\
\hline 1476 & 0.754 & 0.40 & Free & NA \\
1644 & 0.754 & 0.48 & Free & NA \\
1932 & 0.754 & 0.66 & Free & NA \\
2190 & 0.754 & 0.84 & Cavitation & 10.24 \\
2466 & 0.754 & 1.80 & Cavitation & 58.10 \\
2682 & 0.754 & 1.27 & Cavitation & 40.63 \\
\hline
\end{tabular}
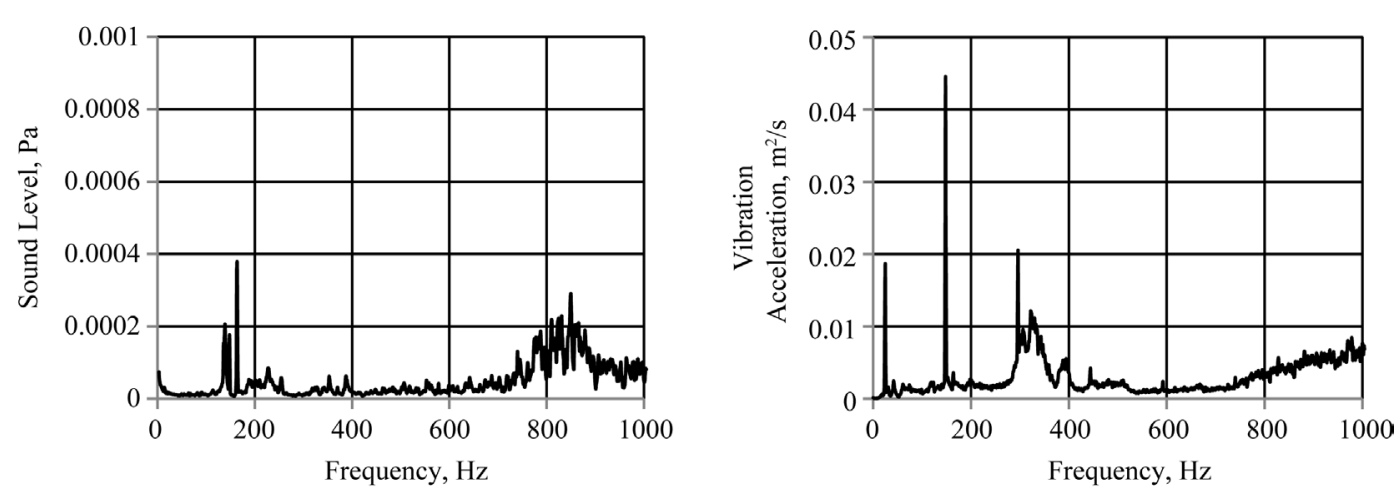

(a)
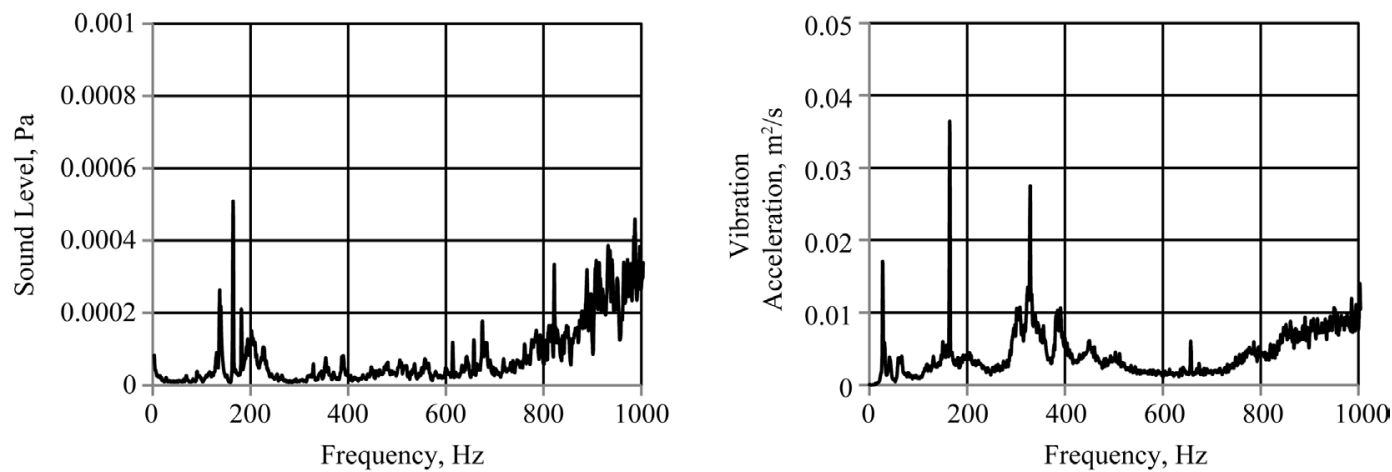

(b)
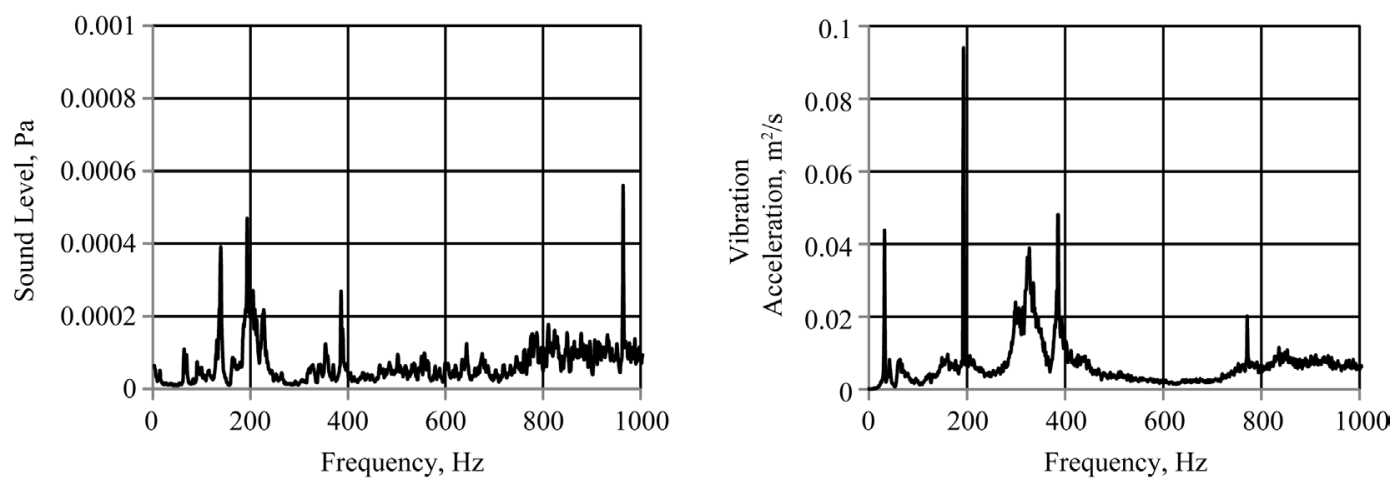

(c)

Figure 3. RMS of vibration acceleration and sound levels for group 1. (a) Vibration acceleration and sound at $1476 \mathrm{rpm}$; (b) vibration acceleration and sound at $1644 \mathrm{rpm}$; (c) vibration acceleration and sound at 1932 rpm. 
sound in the frequency range up to $1000 \mathrm{~Hz}$, for the group 1. Figure 4(a), Figure 4(b), and Figure 4(c) shows the root mean square for both the vibration acceleration and sound in the frequency range up to $1000 \mathrm{~Hz}$, for the group 2.

Analysis of Figure 3 and Figure 4 depend on the follow up of the rotational frequency $(R F=r p m / 60)$, blade passing frequency $(B P F=n \times r p m / 60)$ and second ( $2 B P F=2 \times n \times r p m / 60)$, third (3BPF $=3 \times n \times r$ rpm/60), fourth (4BPF $=4 \times \mathrm{n} \times \mathrm{rpm} / 60$ ) harmonics. Where $n$ is the number of blades $=6$.

At the rotational frequency, both vibration acceleration and sound are taken from Figure 3 and Figure 4 and clearly demonstrated at Figure 5. For group 1 (no cavitation) there are apparent vibration signals at the rotational frequencies corresponding to the rotational speeds, while sound signals completely disappeared. For group 2 (cavitation) there are still vibration signals at the rotational frequencies, while only one sound signal appears at rotational frequency of $36.5 \mathrm{~Hz}$ corresponds to the rotational speed of $2190 \mathrm{rpm}$.

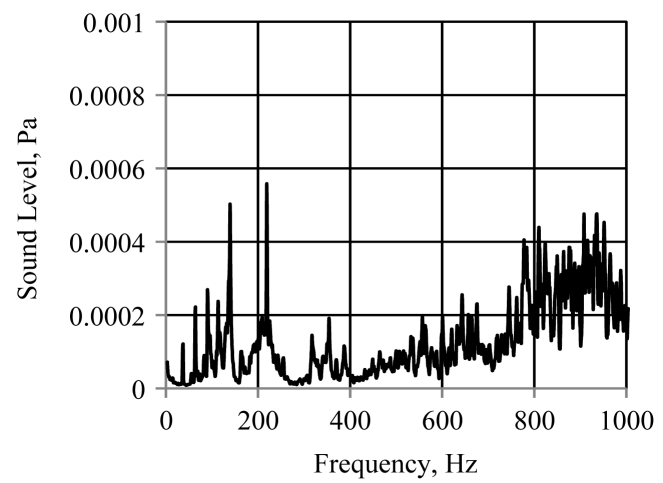

(a)

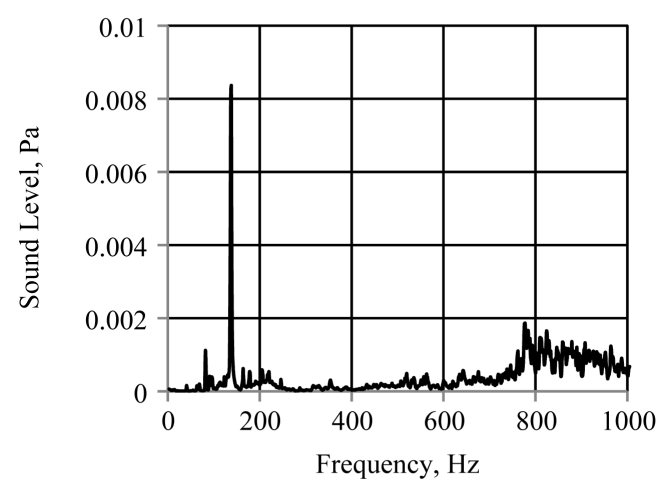

(b)

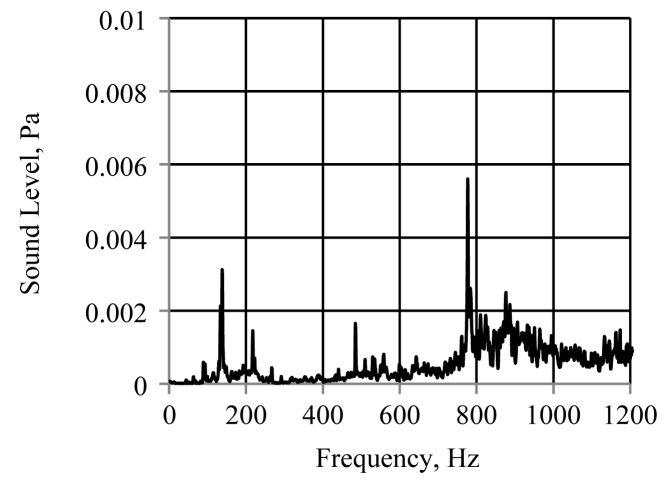

(c)
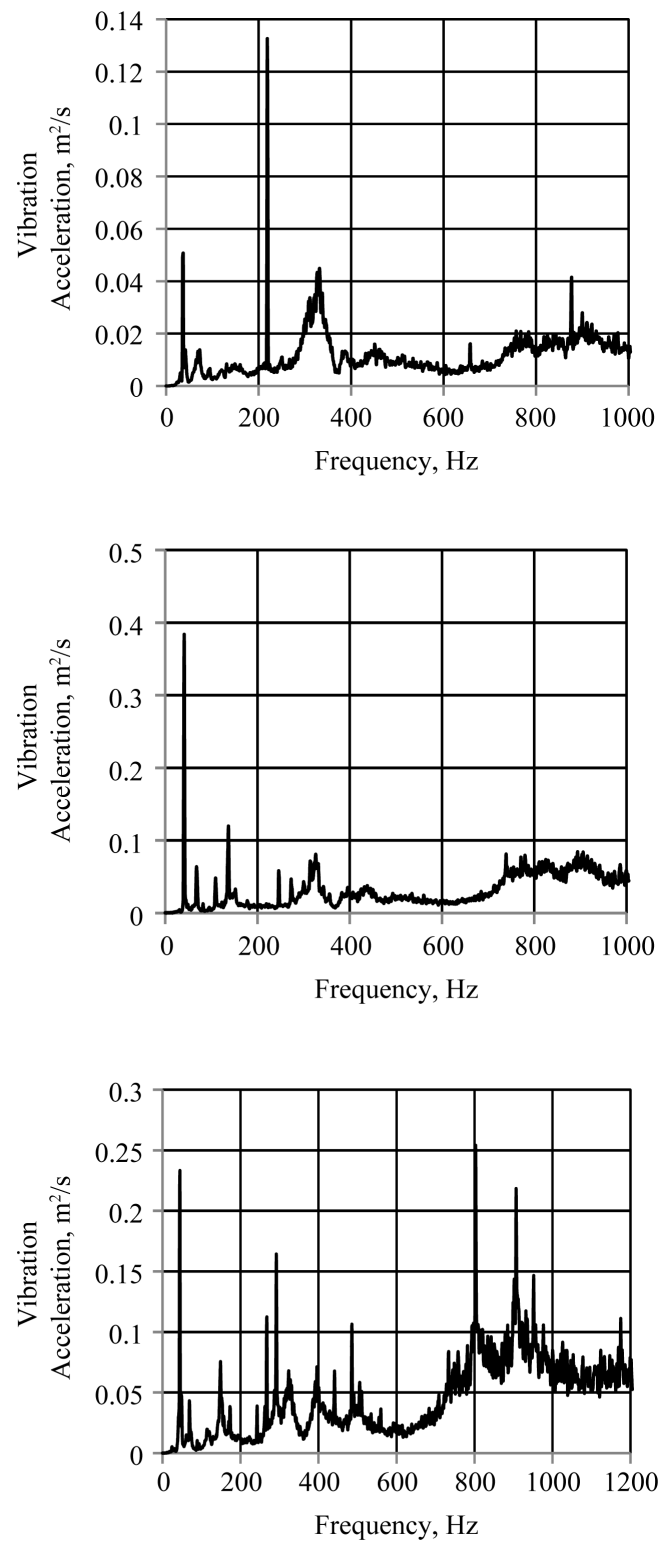

Figure 4. RMS of vibration acceleration and sound levels for group 2. (a) Vibration acceleration and sound at $2190 \mathrm{rpm}$; (b) vibration acceleration and sound at $2466 \mathrm{rpm}$; (c) vibration acceleration and sound at 2682 rpm. 


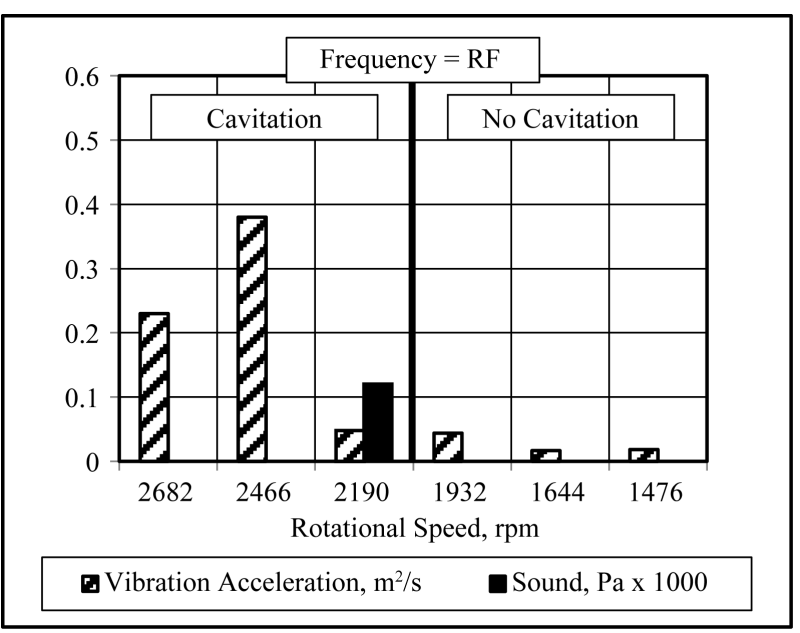

Figure 5. RMS of vibration acceleration and sound levels at the rotational frequency for different rotational speeds.

In case of no cavitation, increasing the rotational speed has no guided trend on the level of the vibration acceleration; this makes the variable speed pump not easy to be monitored. Therefore the variable speed pump may appear high vibration signals at the rotational frequency in the case of no cavitation. In case of cavitation, strengthed vibration signals appear at the rotational frequency with level depends slightly on the rotational speed and greatly on the strength of cavitation. Rotational speed of $2466 \mathrm{rpm}$ has the highest vibration acceleration of $0.38 \mathrm{~m}^{2} / \mathrm{s}$ because it has the highest cavitation strength of $58.1 \%$. Generally; the vibration signals in the case of cavitation are more strengthed than the case for no cavitation at the rotational speed frequencies.

Regarding to Figure 4(a), the sound signal appears at the rotational frequency of $36.5 \mathrm{~Hz}$ corresponds to the rotational speed of $2190 \mathrm{rpm}$ is followed by many high noise signals at near frequencies of $64 \mathrm{~Hz}, 90 \mathrm{~Hz}, 113$ $\mathrm{Hz}$, and $139 \mathrm{~Hz}$. For this reason, one cannot consider the cavitation signs the sound spectrum at the rotational frequency. At the blade passing frequency, both vibration acceleration and sound are taken from Figure 3 and Figure 4 and clearly demonstrated at Figure 6. For group 1 (no cavitation) there are apparent vibration signals at the blade passing frequencies corresponding to the rotational speeds, while sound signals completely disappeared at the rotational speed of $1476 \mathrm{rpm}$ and appear at the rotational speeds of $1644 \mathrm{rpm}$ and $1932 \mathrm{rpm}$. For group 2 (cavitation) there are still vibration signals at the blade passing frequencies, while only one noise signal is appeared at the blade passing frequency corresponds to rotational speed of $2190 \mathrm{rpm}$. In case of no cavitation, as an emphasis for the previous result, increasing the rotational speed has no guided trend on the level of the vibration acceleration.

In case of cavitation, in order of magnitude levels of vibration signals appear at the rotational frequency. It is noted that the levels of vibration acceleration at the blade passing frequency are reverse to that at the rotational frequencies as illustrated in Figure 7. Therefore the cavitation in variable speed pumps is detected by online comparing between the vibration acceleration at the rotational and blade passing frequencies, at which the levels at the rotational frequency are decreased while the levels at the blade passing frequency are increased or vice versa is detected, this is an indication for cavitating pump which is unique and not occurred for the case of no cavitation.

It is noted that the levels of sound at the blade passing frequency of $219 \mathrm{~Hz}$ corresponds to the rotational speed of $2190 \mathrm{rpm}$ is higher than the corresponding one at the rotational frequency, but still the sound signals appear in a random manner.

At the second blade passing frequency, both vibration acceleration and sound are taken from Figure 3 and Figure 4 and clearly demonstrated at Figure 8. For group 1 (no cavitation) there are apparent vibration signals at the second blade passing frequencies corresponding to the rotational speeds, while sound signals completely disappeared at the rotational speed of $1476 \mathrm{rpm}$ and $1644 \mathrm{rpm}$ and appeared at the rotational speeds of $1932 \mathrm{rpm}$. For group 2 (cavitation) both the vibration acceleration and sound signals are disappeared from the spectrum.

At the third blade passing frequency, both vibration acceleration and noise are taken from Figure 3 and Figure 4 and clearly demonstrated at Figure 9. For group 1 (no cavitation) there are no apparent vibration signals at 


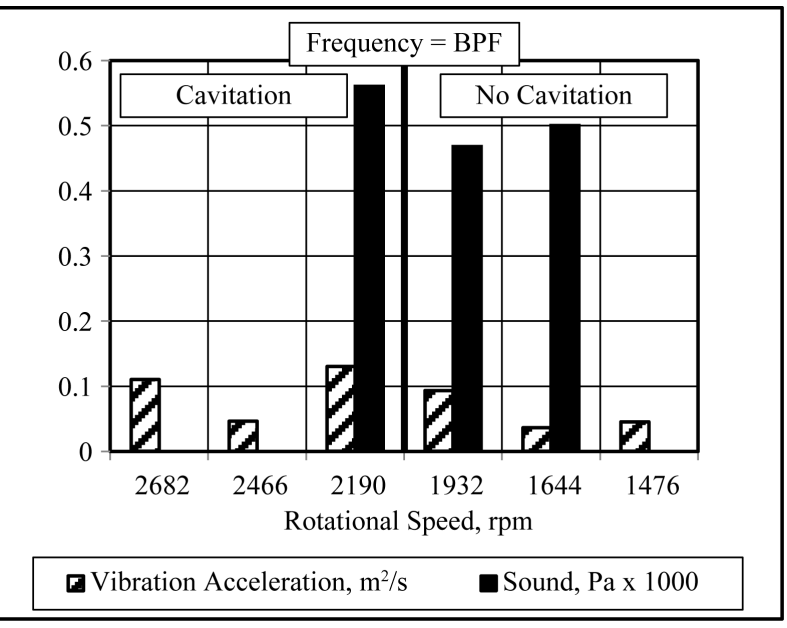

Figure 6. RMS of vibration acceleration and sound levels at the blade passing frequency for different rotational speeds.

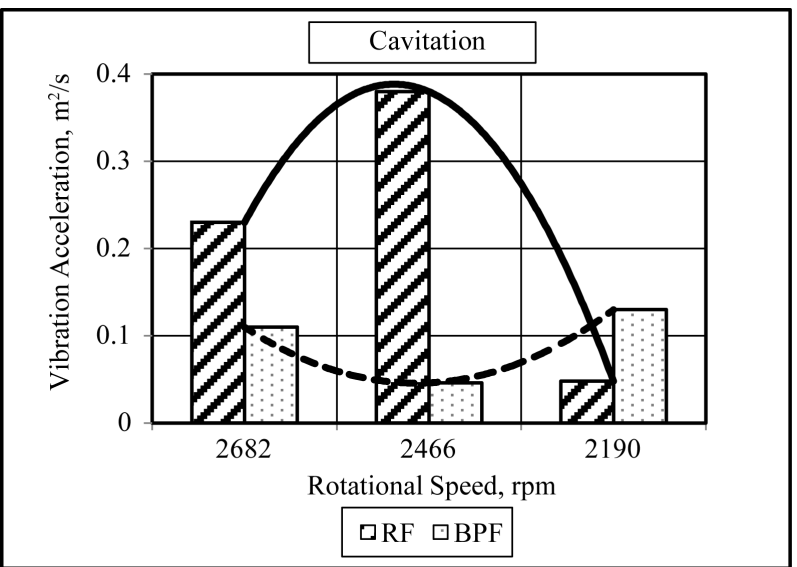

Figure 7. RMS of vibration acceleration levels at the rotational and blade passing frequencies for different rotational speeds at cavitation conditions.

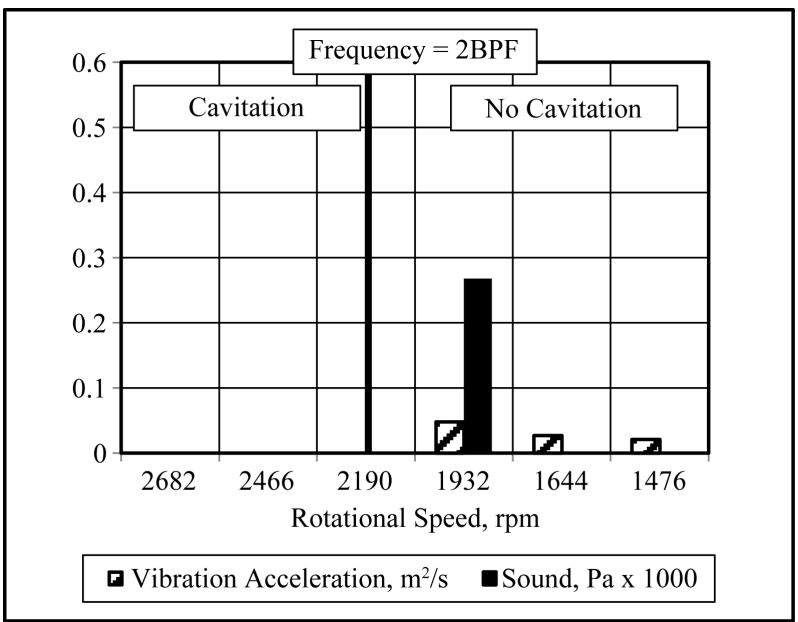

Figure 8. RMS of vibration acceleration and sound levels at the second blade passing frequency for different rotational speeds. 
the third blade passing frequencies corresponding to the rotational speeds, and sound signals completely disappeared.

At the fourth blade passing frequency, both vibration acceleration and sound are taken from Figure 3 and Figure 4 and clearly demonstrated at Figure 10. For group 1 (no cavitation) there is only apparent vibration signal at the fourth blade passing frequency corresponds to the rotational speed of $1932 \mathrm{rpm}$, while a sound signal appears at the rotational speed of $1644 \mathrm{rpm}$. For group 2 (cavitation) there is still a vibration signal at the fourth blade passing frequency corresponding to the rotational speed of $2190 \mathrm{rpm}$, while sound signals are disappeared at the fourth blade passing frequency.

Comparing Figure 5 to Figure 10, the noise signals at the discrete frequencies appear at a random manner. For this reason, one cannot count on the detection of pump cavitation by sound surveying at discrete frequencies. The vibration signals at the second, third, and fourth blade passing frequencies appear at a random manner. For this reason, one cannot count on the detection of pump cavitation by vibration surveying at the harmonics of the blade passing frequency.

For centrifugal pump operates under cavitation conditions, therefore Figure 5 to Figure 7 reveals that one can count on the vibration measurements at the rotational and blade passing frequencies.

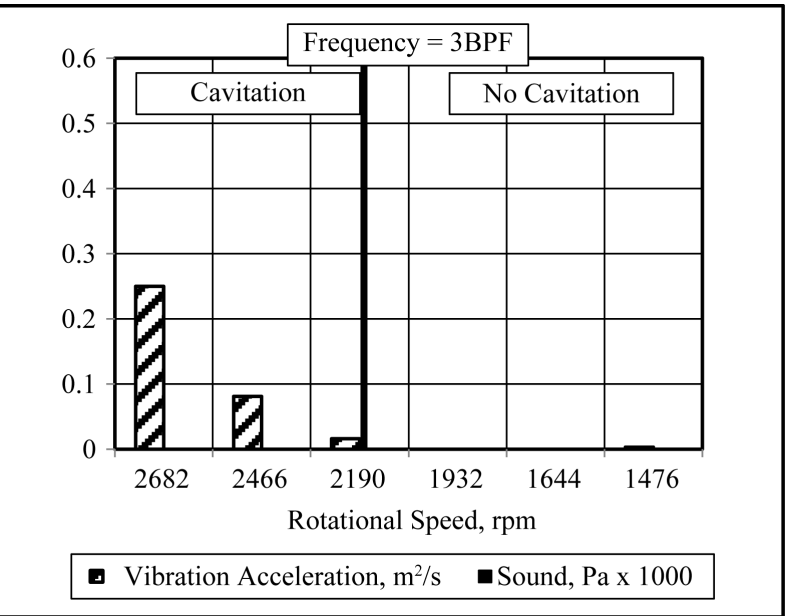

Figure 9. RMS of vibration acceleration and sound levels at the third blade passing frequency for different rotational speeds.

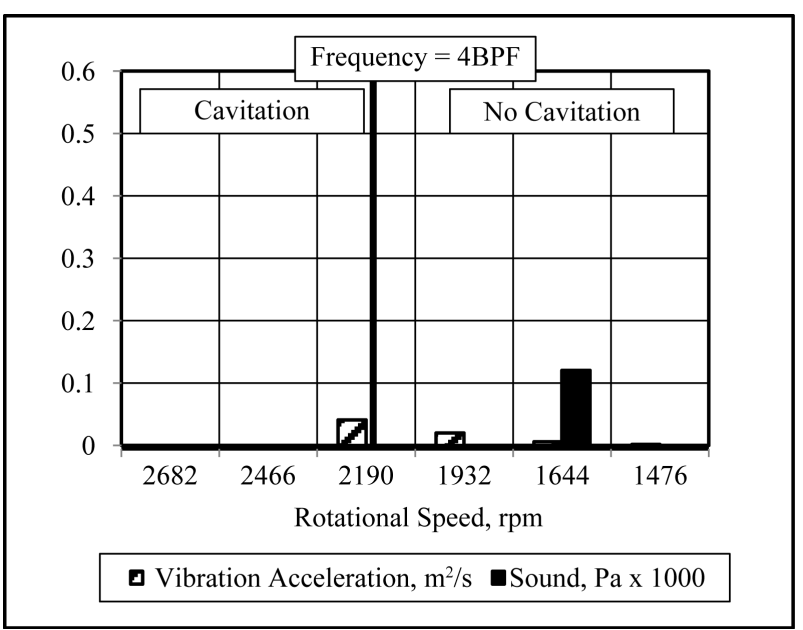

Figure 10. RMS of vibration acceleration and sound levels at the fourth blade passing frequency for different rotational speeds. 
Regarding to the vibration acceleration at the rotational and blade passing frequencies at variable speed in the case of cavitation as in Figure 5 and Figure 6, the level of vibration is composed from two parameters; the first is the vibration level due to the impact of the rotational speed while the second is the vibration level due to the effect of the cavitation strength. To the author knowledge no published data about the effect of variable speed at constant cavitation strength and pump discharge, which is recommended for future work.

\section{Conclusion}

For variable speed pump discharges $780 \mathrm{~L} / \mathrm{h}$ with $\mathrm{NPSH}_{\mathrm{A}}$ of $0.754 \mathrm{~m}$ rotates at speeds of $1476 \mathrm{rpm}, 1644 \mathrm{rpm}$, $1932 \mathrm{rpm}, 2190 \mathrm{rpm}, 2466 \mathrm{rpm}$, and $2682 \mathrm{rpm}$ it is concluded that:

1) The occurrence of cavitation depends on the rotational speed.

2) The sound signals in both no cavitation and cavitation conditions appear in random manner.

3) Surveying the vibration and sound spectra at the second, third, and fourth blade passing frequencies reveals no indications or phenomenon associated with the cavitation at variable speeds.

4) For cavitation detection in pump, it is recommended to survey the vibration spectra at the rotational and blade passing frequencies simultaneously.

5) Comparing between the vibration acceleration at the rotational and blade passing frequencies, at which the levels at the rotational frequency are decreased while the levels at the blade passing frequency are increased or vice versa is detected, this is an indication for cavitating pump which is unique and not occurred for the case of no cavitation.

\section{References}

[1] Al-Arabi, A.A.B., Selim, S.M.A., Saidur, R., Kazi, S.N. and Duffy, G.G. (2011) Detection of Cavitation in Centrifugal Pumps. Australian Journal of Basic and Applied Sciences, 5, 1260-1267.

[2] Cernetic, J. (2009) The Use of Noise and Vibration Signals for Detecting Cavitation in Kinetic Pumps. Proceedings of the Institution of Mechanical Engineers, Part C: Journal of Mechanical Engineering Science, 223, 1645-1655. http://dx.doi.org/10.1243/09544062JMES1404

[3] Birajdar, R., Patil, R. and Khanzode, K. (2009) Vibration and Noise in Centrifugal Pumps-Sources and Diagnosis Methods. 3rd International Conference on Integrity, Reliability and Failure. Porto. (Paper Ref: S1163_P0437).

[4] Alfayez, L., Mba, D. and Dyson, G. (2005) The Application of Acoustic Emission for Detecting Incipient Cavitation and the Best Efficiency Point of a 60 KW Centrifugal Pump: Case Study. NDT\&E International, 38, 354-358. http://dx.doi.org/10.1016/j.ndteint.2004.10.002

[5] Cernetic, J., Preselj, J. and Cudina, M. (2008) Use of Noise and Vibration Signal for Detection and Monitoring of Cavitation in Kinetic Pumps. Acoustics 08 Paris, June 29-July 4 2008, 2199-2204.

[6] Jan, C. and Cudina. M. (2012) Cavitation Noise Phenomena in Centrifugal Pumps. 5th Congress of Alps-Adria, Acoustics Association, 12-14 September 2012, Petrčane.

[7] Albraik, A., Althobiani, F., Gu, F. and Ball, A. (2012) Diagnosis of Centrifugal Pump Faults Using Vibration Methods. Journal of Physics: Conference Series, 364, Article ID: 012139. http://dx.doi.org/10.1088/1742-6596/364/1/012139

[8] Suhane, A. (2012) Experimental Study on Centrifugal Pump to Determine the Effect of Radial Clearance on Pressure Pulsations, Vibrations and Noise. International Journal of Engineering Research and Applications (IJERA), 2, 18231829.

[9] Gupta, S., Chouksey, V.K. and Srivastava, M. (2013) Online Detection of Cavitation Phenomenon in a Centrifugal Pump Using Audible Sound. ITSI Transactions on Electrical and Electronics Engineering (ITSI-TEEE), 1, 103-107.

[10] Hamomd, O., Tian, X.G., Chen, Z., Albraik, A., Gu, F.S. and Ball, A. (2014) A New Method of Vibration Analysis for The Diganosis of Impeller in a Centrifugal Pump. The 21st International Congress on Sound and Vibration, 13-17 July 2014, Beijing.

[11] Ramadevi, R. (2014) Classification of Vibration Signal to Detect Pump Cavitation Using Discrete Wavelet Transform. International Journal of Computer Applications (0975-8887), 93, 36-39.

[12] Yan, Z.L., Liu, J., Chen, B., Cheng, X.B. and Yang, J. (2015) Fluid Cavitation Detection Method with Phase Demodulation of Ultrasonic Signal. Applied Acoustics, 87, 198-204. http://dx.doi.org/10.1016/j.apacoust.2014.07.007

[13] BPMA the Pump Industry Association. Variable Speed Driven Pumps-Best Practice Guide. http://www.bpma.org.uk/filemanager_net/files/gpg344_variable_speed_best_practice_for_pumps.pdf 